\title{
Inhibition of Euchromatic Histone Lysine Methyltransferase 2 (EHMT2) Suppresses the Proliferation and Invasion of Cervical Cancer Cells
}

\author{
Guoqiang Chen $^{\mathrm{a}}$ Xiaqing Yu ${ }^{\mathrm{b}}$ Min Zhang ${ }^{\mathrm{c}}$ Aiwen Zheng ${ }^{\mathrm{d}}$ Zhennan Wang ${ }^{\mathrm{a}}$ \\ Yufang Zuo ${ }^{a}$ Qilian Liang ${ }^{\text {a }}$ Danxian Jiang ${ }^{\text {a }}$ Yaqing Chen $^{d}$ Lingqin Zhao $^{d}$ \\ Liyuan Jiang $^{c}$ Dan Li ${ }^{\mathrm{b}}$ Sihai Liao ${ }^{\mathrm{a}}$ \\ a Department of Oncology, Affiliated Hospital of Guangdong Medical University, Zhanjiang, China; \\ ${ }^{b}$ Department of Nuclear Medicine, Shanghai Tenth People's Hospital, Shanghai, China; \\ 'Department of Obstetrics, Hangzhou Obstetrical and Gynecological Hospital, Hangzhou, China; \\ ${ }^{\mathrm{d}}$ Department of Gynecology, Zhejiang Cancer Hospital, Hangzhou, China
}

\section{Keywords}

Cervical cancer cells $\cdot$ EHMT2 $\cdot$ Histone methyltransferase · Invasion · Proliferation

\begin{abstract}
EHMT2 (euchromatic histone lysine methyltransferase 2), a histone methyltransferase, has been shown to be involved in multiple human cancers. In this study, we determined mRNA and protein expression of EHMT2 in cervical cancer cells and normal cervical epithelial cells. EHMT2 was inhibited with short hairpin RNA (shEHMT2) in cervical cancer cells. Cell viability, colony proliferation, apoptosis, adhesion, and invasion assays and Western blot were performed to assess the function of EHMT2. As a result, EHMT2 was upregulated in human cervical cancer cells compared to normal cervical epithelial cells. Suppression of EHMT2 expression impairs cell proliferation and induces apoptosis. Furthermore, EHMT2 silencing inhibited cell adhesion and invasion. Finally, knockdown of EHMT2 resulted in a reduction of the expression of the tumorigenic proteins $\mathrm{Bcl}-2, \mathrm{Mcl}-1$, and Survivin and in an increase in the expression of the anti-malignant protein E-cadherin. In conclusion, our data suggest that EHMT2 plays a key role in cell proliferation and metastatic capacity in cervical cancer cells and could serve as a potential therapeutic target.

(c) 2019 S. Karger AG, Basel
\end{abstract}

๑ 2019 S. Karger AG, Basel
Cervical cancer is the most common malignant tumor in women all over the world and maintains the second highest incidence and elevated mortality rate in China [Ili et al., 2011]. Meanwhile, cervical cancer patients become younger, and cervical cancer threatens the life quality of women significantly [Arbyn et al., 2011]. Because of a lack of early detection by systematic and effective large-scale screening, most patients miss the right time to do surgery [Shen et al., 2014]. However, the outcomes of radiotherapy and chemotherapy are various. Thus, cervical cancer is a worldwide health issue [Wang and Qiao, 2015].

It was reported that acquired gemcitabine resistance is associated with hyper-expression of euchromatin histone lysine methyltransferase 2 (EHMT2), also known as G9a. Inhibition of the histone methyltransferase can reverse gemcitabine resistance in cervical cancer cells [Candelaria et al., 2012]. This research inspired us to study the role of EHMT2 in cervical cancer. EHMT2 is the second member of a conserved protein family which can demethylate histone 3 at lysine 9 in euchromatin. The protein has been shown to be involved in numerous physiologic processes, including DNA methylation [Auclair et al., 2016], gene silencing in embryogenesis, and cellular differentiation [Kramer, 2016]. It has also been shown to modulate nu-

G.C., X.Y., and M.Z. contributed equally to this work.

\section{KARGER}

E-Mail karger@karger.com

www.karger.com/cgr
Sihai Liao

Department of Oncology

Affiliated Hospital of Guangdong Medical University

No. 57, City People's Road South, Zhanjiang, Guangdong, 524002 (China)

E-Mail liaoshkxy@sina.com 
merous pathologic processes and has been implicated in a large number of malignancies [Cho et al., 2011; Ding et al., 2013; Heo et al., 2013].

A previous study showed a higher expression of EHMT2/G9a in invasive cervical cancer than in cancer precursors or normal epithelium cells and that overexpression of EHMT2/G9a increases the proliferation, migration, and invasion in cervical cancer cell lines [Chen et al., 2017]. Increased expression of EHMT2 has been shown to be associated with higher stage, higher grade, and more peritoneal metastases in ovarian cancer [Hua et al., 2014]. EHMT2 expression levels also directly predict the prognosis in lung cancer [Chen et al., 2010]. Esophageal squamous cell tumors that stained more intensely for EHMT2 were of higher histological grade, demonstrated a greater depth of invasion, more lymph node metastases, and were subsequently of a higher stage than less intensely staining tumors [Zhong et al., 2015]. Additionally, patients with esophageal squamous cell carcinomas that overexpressed EHMT2 had a significantly reduced survival rate [Zhong et al., 2015]. Further, increased expression of EHMT2 has been shown to inversely correlate with a response to interferon therapy in CML, suggesting that the higher the EHMT2 expression, the more resistant the leukemia is to chemotherapeutics [Loh et al., 2014]. Thus, in addition to serving as a possible therapeutic target, EHMT2 might also serve as a diagnostic marker in the initial workup of a cervical mass. In order to provide evidence to support the potential role of EHMT2 in cervical cancer, an in vitro study on inhibiting the expression of EHMT2 in cervical cancer is required for validation.

Our study aimed to observe the effect of inhibiting EHMT2 on proliferation and metastasis in human cervical cancer cells in vitro.

\section{Materials and Methods}

\section{Cell Culture}

Four human cervical cancer cell lines (Caski, C-33A, SiHa, and $\mathrm{HeLa}$ ) and an immortalized cervical epithelial cell line (NC104) were obtained from the Cell Bank of the Shanghai Institute of Biochemistry and Cell Biology, Chinese Academy of Sciences (Shanghai, China). HEK293T cells were stored in our laboratory. All cells were cultivated in Dulbecco's modified Eagle's medium (DMEM, Invitrogen, USA) supplemented with $10 \%$ fetal bovine serum (FBS, Gibco, USA), 100 units/mL penicillin, and $100 \mu \mathrm{g} / \mathrm{mL}$ streptomycin at $37^{\circ} \mathrm{C}$ in a humidified atmosphere containing $5 \% \mathrm{CO}_{2}$.

Quantitative Real-Time PCR Analysis

Quantitative real-time PCR (qPCR) analysis was performed for detecting mRNA levels in the cells. Total RNA was extracted from Caski, C-33A, SiHa, HeLa, and NC104 using the Trizol reagent
(Qiagen, Germany) and treated with RNase-free DNaseI (Promega, USA). RNA was reverse transcribed to cDNA using PrimeScript RRT Master Mix Perfect Real Time (Takara, Japan) and amplified using a PCR kit (Qiagen). The EHMT2 primers were: upstream primer: $5^{\prime}$-TCCAATGACACATCTTCGCTG-3' ${ }^{\prime}$; downstream primer: $5^{\prime}$-CTGATGCGGTCAATCTTGGG-3'. The GAPDH primers were: upstream primer: $5^{\prime}$-CTGGGCTACACTGAGCACC-3'; downstream primer: 5'-AAGTGGTCGTTGAGGGCAATG-3'. Amplification and detection were performed using an ABI 7500 Real-Time PCR System (Applied Biosystems, USA). The PCR reactions were performed in duplicate and repeated 3 times.

\section{Plasmid Construction and Lentivirus Infection}

To inhibit the expression of EHMT2, the short hairpin RNA (shRNA) targeting EHMT2 and the scramble control shRNA were synthesized and cloned into pLKO.1-TRC (Addgene: 10878, USA) by Zhongqing Biotechnology company (Suzhou, Jiangsu, China) to generate recombinant plasmids. The sequence of the shRNA targeting EHMT2 was 5'-CCGGCTCCAGGAATTTAACAAGATTCTCGAGAATCTTGTTAAATTCCTGGAGTTTTTG-3' and the sequence of the scramble control shRNA was $5^{\prime}$-CCGGCCTAAGGTTAAGTCGCCCTCGCTCGAGCGAGGGCGACTTAACCTTAGGTTTTTG-3'.

The lentiviruses were produced by co-transfecting the shRNAEHMT2 (or scramble control shRNA), pCMVdeltaR8.91, and pMD2.G into HEK293T cells using X-tremeGENE HP DNA Transfection Reagent (Roche) according to the manufacturer's recommendations. Lentiviral particles were harvested by collecting media from HEK293T cells. The human cervical cancer cell lines with the high expression level of EHMT2 ( $\mathrm{SiHa}$ and HeLa) were infected with the lentiviral particles, and stably silenced cell lines and the control cell lines were obtained with $2 \mu \mathrm{g} / \mathrm{mL}$ puromycin (Sigma, USA). Finally, the knockdown of EHMT2 by shRNA (shEHMT2) was confirmed by Western blot and compared to the control group treated by scramble control shRNA.

\section{Cell Growth Assay}

Cell proliferation was assessed by Cell Counting Kit-8 (CCK-8, Dojindo Laboratories, Kumamoto, Japan) assay. Briefly, cells were harvested $24 \mathrm{~h}$ after infection by lentivirus. The infected cells were seeded on a 96-well microplate at a density of $3 \times 10^{3}$ cells per well and were cultured for 24,48 , and $72 \mathrm{~h}$. Then, $10 \mu \mathrm{L}$ of CCK- 8 solution was added to each well and incubated at $37^{\circ} \mathrm{C}$ for an additional $3 \mathrm{~h}$. Optical density (OD) was determined at a wavelength of $450 \mathrm{~nm}$.

\section{Colony Formation Assay}

Cells were mixed with $0.3 \%$ soft agar (Sigma) and culture media and seeded in 6-well plates (1,000 cells per well) with a solidified bottom layer consisting of $0.6 \%$ soft agar and culture media. After 20 days of treatment, micrographs of cell colonies were taken using inverted microscopy. Then, the cells were stained with MTT, and pictures were taken using a scanner (Epson, USA).

\section{Flow Cytometry Analysis}

Cells were washed twice with ice-cold PBS and were resuspended in staining buffer at a concentration of $1 \times 10^{6}$ cells $/ \mathrm{mL}$. We performed Annexin V and PI staining using the Annexin V-FITC Apoptosis Detection Kit (BD Biosciences, USA) according to the 
manufacturer's instructions. Stained cells were immediately analyzed by the BD LSRII Flow Cytometer.

\section{Cell Adhesion Assay}

To analyze the attachment of the cervical cancer cells to fibronectin, which is an extracellular matrix component, we conducted a cell adhesion assay. Firstly, $1 \times 10^{5}$ cells were harvested and resuspended in culture medium and then transferred to a 24-well plate coated with $100 \mu \mathrm{g}$ fibronectin (Sigma-Aldrich) that was coated with matrix protein $\left(40 \mu \mathrm{g} / \mathrm{mL}\right.$ type I collagen or $2 \mu \mathrm{g} / \mathrm{cm}^{2}$ fibronectin in PBS) at room temperature for $1 \mathrm{~h}$ and washed twice with PBS to remove the non-adherent cells. Then, the adhesion of cells was measured by CCK- 8 in a microplate reader (Multiskan MK3; Thermo Fisher Scientific, USA) at $450 \mathrm{~nm}$ to obtain an OD value. The adhesion of cells was related to the control set at $100 \%$.

\section{Cell Invasion Assay}

Invasion assays were performed in a modified Boyden chamber (NeuroProbe, USA). The cells were resuspended in serum-free medium and placed into the upper chamber at $4 \times 10^{4} /$ well with an $8-\mu \mathrm{m}$ microporous membrane (Costar, Corning Inc, USA) coated with Matrigel (Bioscience, USA). Medium with 20\% FBS was added to the lower chamber and was incubated at $37^{\circ} \mathrm{C}$ for $24 \mathrm{~h}$. Then the migrated cells on the lower surface were fixed with methanol and stained with hematoxylin and counted in 3 random high-power fields $(\times 200)$ in each well.

\section{Western Blot Analysis}

Total cellular proteins were lysed in $300 \mu \mathrm{L}$ lysis buffer containing $1 \mathrm{~mm}$ phenylmethanesulfonyl fluoride (Sigma) and $1 \times$ proteasome inhibitor (Sigma). A total of $20 \mu \mathrm{g}$ of protein were loaded onto $10 \%$ SDS polyacrylamide gels and transferred onto PVDF membranes (Millipore, Germany). Western blotting was performed using primary antibodies against EHMT2 (1:500, Abgent, USA), glyceraldehyde 3-phosphate dehydrogenase (GAPDH, 1:5,000 dilution in 5\% milk, Santa Cruz), E-cadherin, Bcl-2, Mcl-1, and Survivin (1:1,000, Cell Signaling Technology, USA), and secondary goat anti-rabbit antibodies (1:4,000, PerkinElmer, USA). Autoradiographs were scanned and analyzed semiquantitatively. All of the shown images are from a single experiment that is representative of at least 3 separate experiments.

\section{Statistical Analysis}

Data are expressed as the mean \pm SD of 3 distinct attempts. Significance was determined using a Student $t$ test. Data were analyzed with GraphPad Prism software (version 7.00). A value of $p<0.05$ was considered as statistically significant.

\section{Results}

\section{Overexpression of EHMT2 in Cervical Cancer Cells}

Compared to Cervical Epithelial Cells

Expression levels of EHMT2 were identified in multiple human cervical cancer cell lines and human cervical epithelial cell line by qRT-PCR and Western blot. Expression of EHMT2 mRNA was significantly higher in the

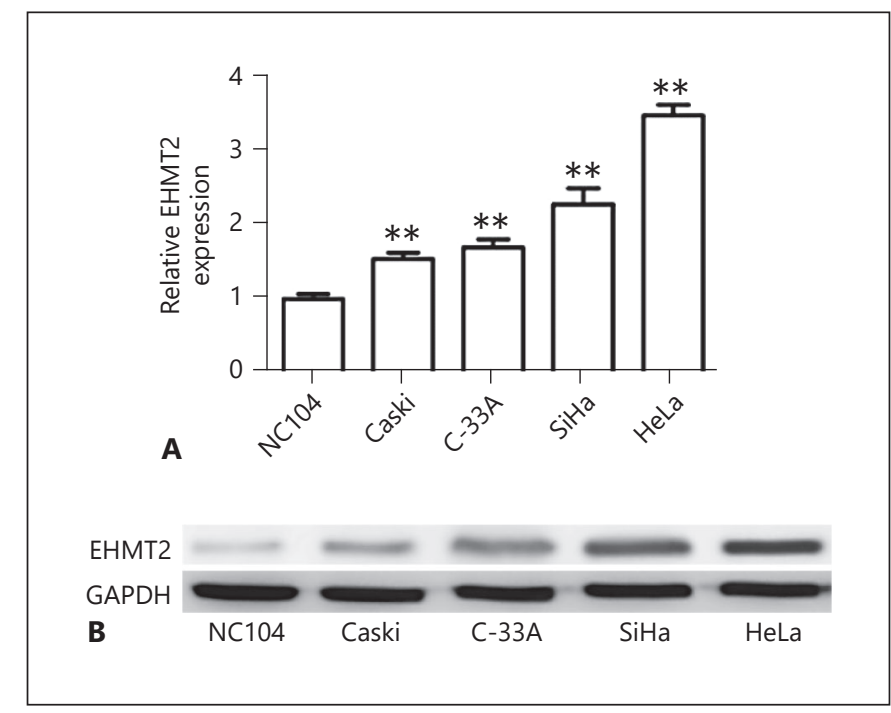

Fig. 1. EHMT2 is overexpressed in malignant cervical cancer cells compared to normal cervical epithelial cells. A Quantitative realtime PCR analysis demonstrated relative EHMT2 mRNA expression in a cervical epithelial cell line (NC104) and multiple human cervical cancer cell lines (Caski, C-33A, SiHa, and HeLa). The GAPDH expression level served as an endogenous reference. Significance in each cancer cell line was determined by using a Student $t$ test compared to the normal cell line. ${ }^{* *} p<0.01$. B Western blot demonstrated relative EHMT2 protein expression in a cervical epithelial cell line (NC104) and multiple human cervical cancer cell lines (Caski, C-33A, SiHa, and HeLa). Total GAPDH levels served as loading controls.

cervical cancer cell lines (Caski, C-33A, SiHa, and HeLa) than in the normal cervical epithelial cell line (NC104) $(p<0.01)$ (Fig. 1A). As we expected, EHMT2 protein expression was higher in the cervical cancer cell lines (Caski, C-33A, SiHa, and HeLa) than in NC104 as demonstrated by Western blot analysis (Fig. 1B). These results suggested that EHMT2 might act as an oncogene in cervical cancer.

\section{Knockdown of EHMT2 Expression Impairs \\ Proliferation of Cervical Cancer Cells}

Cell proliferation is an important measure characteristic for tumor aggressiveness. To assess the effect of EHMT2 in cervical cancer cells, endogenous EHMT2 expression was knocked down by lentiviral vectors in $\mathrm{SiHa}$ and HeLa cells. The efficacy of shEHMT2 silencing was confirmed using Western blot: cells treated with shEHMT2 demonstrated a reduced expression of EHMT2 (Fig. 2A).

Cell viability of cervical cancer cells treated with shEHMT2 was assessed. SiHa cells that were treated with 


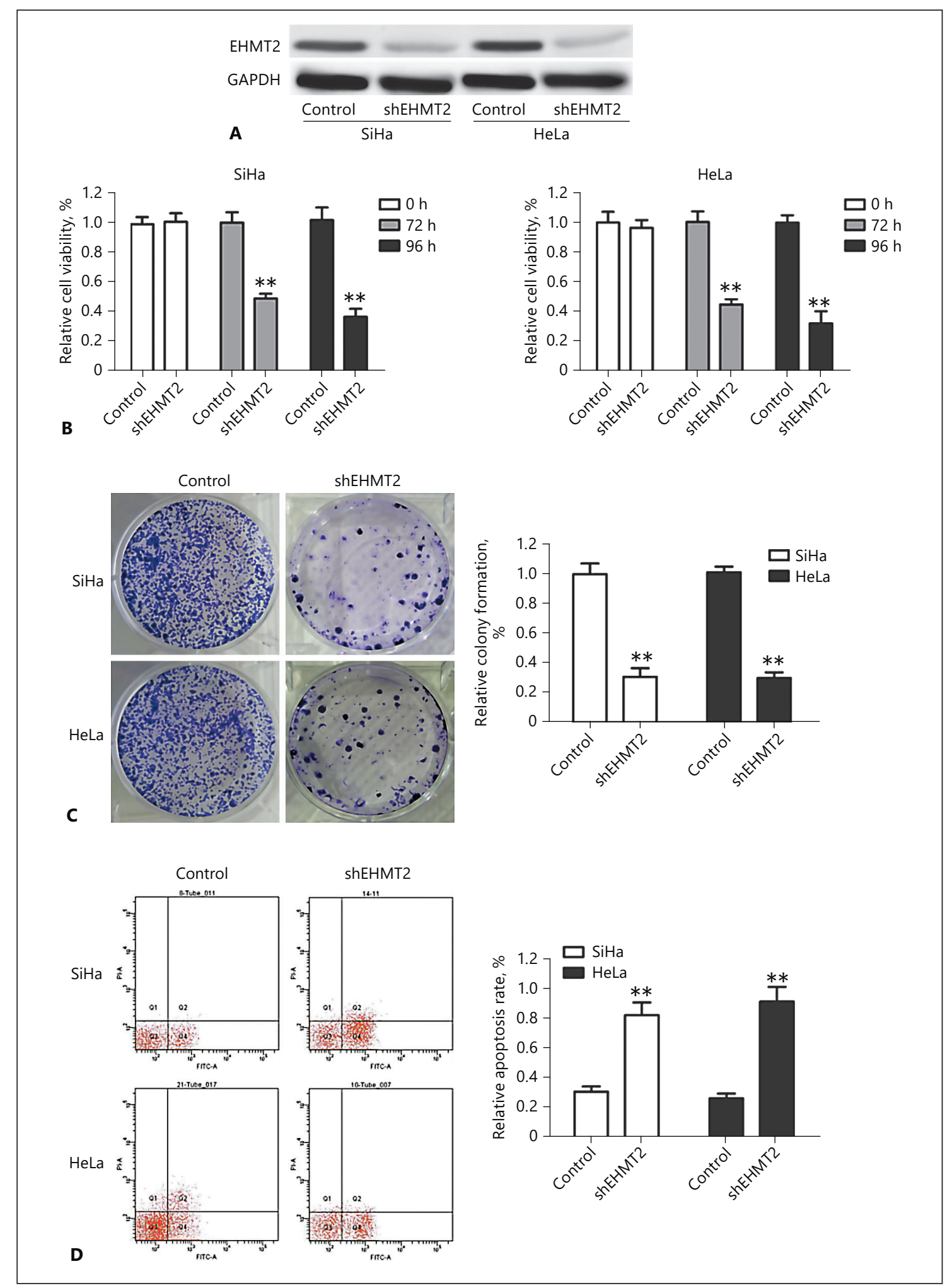

Fig. 2. Reduction of EHMT2 expression impairs the growth of human cervical cancer cells. A Western blot demonstrated the efficacy of the interference RNA shEHMT2 in 2 cervical cancer cell lines. B Reduction of EHMT2 expression reduced relative cell vi- ability at 72 and $96 \mathrm{~h}$ in 2 cervical cancer cell lines. C Reduction of EHMT2 expression reduced colony proliferation in 2 cervical cancer cell lines. D Reduction of EHMT2 expression increased the rate of apoptosis in 2 cervical cancer cell lines. ${ }^{* *} p<0.01$. 
Fig. 3. Reduction of EHMT2 expression impairs the metastatic capacity of cervical cancer cells. A Reduction of EHMT2 expression reduced the adhesive capability of 2 cervical cancer cell lines. B Reduction of EHMT2 expression reduced the invasive capacity of 2 cervical cancer cell lines. ** $p<0.01$.

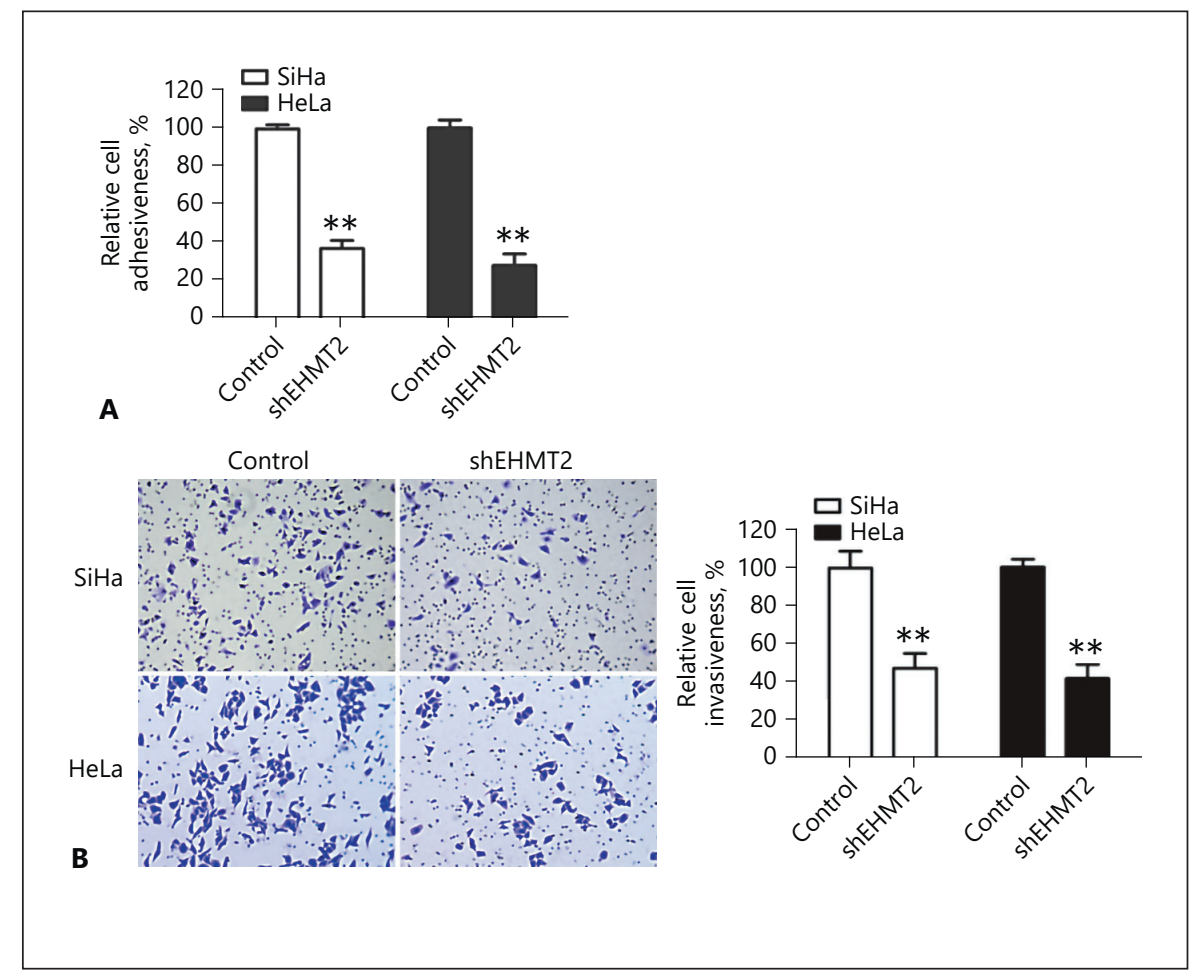

shEHMT2 had significantly reduced cell viability at both 72 and $96 \mathrm{~h}$ (Fig. 2B, left). Also, HeLa cells that were treated with shEHMT2 had significantly reduced cell viability at both 72 and $96 \mathrm{~h}$ compared to control cells (Fig. 2B, right).

Similarly, the ability to colony formation was then examined to test the proliferative capacities of the cells. Both $\mathrm{SiHa}$ and HeLa cells treated with shEHMT2 formed fewer colonies compared to control SiHa cells (Fig. 2C). In addition, cell apoptosis was assayed by flow cytometry. Both SiHa $(p<0.01)$ and HeLa $(p<0.01)$ cells that were treated with shEHMT2 demonstrated significantly higher rates of apoptosis compared to control $\mathrm{SiHa}$ and $\mathrm{HeLa}$ cells that were not treated with the interference RNA, respectively (Fig. 2D).

\section{Reduction in EHMT2 Expression Impairs the Adhesion and Invasion Capacities of Cervical Cancer Cells}

The migratory and invasive capacity of cells could reflect the ability of tumor cell metastasis, which is a vital component of tumor aggressiveness. Therefore, we performed a cell adhesion assay to evaluate migratory ability of the cells affected by inhibition of EHMT2. SiHa and HeLa cells treated with shEHMT2 had significantly lower adhesive ratios than control cells $(p<0.01)$ (Fig. 3A). Moreover, a cell invasion assay was conducted to observe the alteration of invasive ability affected by knockdown of EHMT2. A significantly smaller percentage of SiHa cells treated with shEHMT2 were found to be invasive $(p<0.01)$ compared to the percentage of control cells (Fig. 3B). Similarly, a significantly smaller percentage of HeLa cells treated with shEHMT2 were found to be invasive $(p<0.01)$ (Fig. 3B).

\section{Reduction of EHMT2 Expression Results in Reduced} Expression of Tumorigenic Proteins and Increased Expression of Anti-Malignancy Proteins

To examine the downstream effects on protein expression of EHMT2 inhibition in cervical cancer cells, some proteins involved in cellular proliferation and metastasis were selected for the following study. We found underexpression of Survivin, Mcl-1, and Bcl-2 in EHMT2-knockdown $\mathrm{SiHa}$ and HeLa cell lines as compared to control cells (Fig. 4). Furthermore, knockdown of EHMT2 dramatically increased E-cadherin expression both in $\mathrm{SiHa}$ and HeLa cells (Fig. 4). Finally, our results showed that EHMT2 might mediate the expression of Survivin, Mcl-1, Bcl-2, and E-cadherin. 


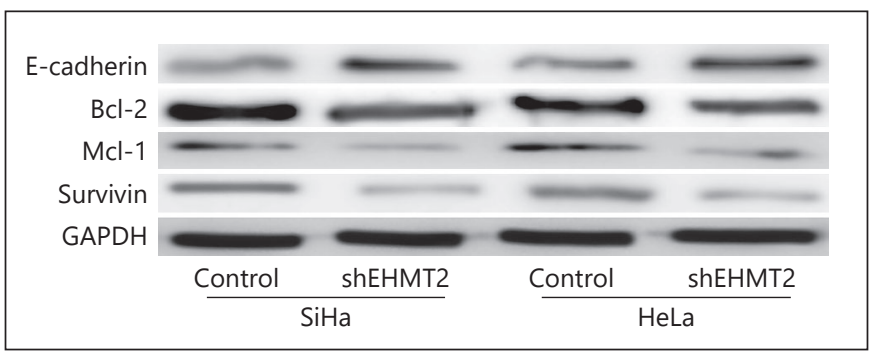

Fig. 4. Reduction of EHMT2 expression results in reduced expression of tumorigenic proteins (Bcl-2, Mcl-1, and Survivin) and increased expression of anti-malignancy protein (E-cadherin).

\section{Discussion}

Generally speaking, EHMT2 is overexpressed in cancer cells compared to normal cells in human cervical cancer cell lines, which has been reported in a previous study [Chen et al., 2017]. The pattern of overexpression is seen in numerous malignancies and indicates that the protein may act via common mechanisms. It has been suggested that it is EHMT2's activation of the serine-glycine biosynthetic pathway that is responsible for its tumorigenic effects, whereby increased EHMT2 expression activates serine and serine-related molecule synthesis, thereby blocking apoptosis [Ding et al., 2013]. Thus, overproduction of EHMT2 hampers native rates of apoptosis and pushes cells towards malignancy. Correspondingly, inhibition of EHMT2 might reduce serine and serine-related molecule synthesis, causing cell death and normalizing rates of apoptosis. Moreover, studies showed that EHMT2/G9a promoted the expression of angiogenic factors, and inhibition of EHMT2 impaired angiogenesis and induced cell cycle arrest in G1 phase [Chen et al., 2017; Wojtala et al., 2018]. These previous findings illustrate the important role of EHMT2 in promoting tumor aggressiveness.

Other research has shown that interaction between EHMT2 and the tumor suppressor gene TP53 may play a role in malignancy [Heo et al., 2013]. EHMT2-mediated p53 inhibition is theorized to occur via direct dimethylation of the protein [Liu et al., 2011]. It should be noted that p53 is also thought to be methylated by EHMT1. Generally, p53 is active in arresting the cell cycle, but in the presence of EHMT2 overexpression, p53 becomes dimethylated and is thus inactivated. EHMT2 also methylates the promoter of SIAH1, an essential gene in the induction of apoptosis, thereby preventing its transcription [Cho et al., 2011]. Without SIAH1, apoptosis rates drop dramatically. However, normal apoptosis resumes when EHMT2 is degraded with interference RNA.

Similarly, EHMT2 has also been shown to methylate the E-cadherin promoter, blocking the transcription of E-cadherin mRNA, while subsequent EHMT2 knockdown restores E-cadherin levels [Dong et al., 2012]. In lung cancer cells, EHMT2 has been shown to potentially interact with another cell adhesion protein, Ep-CAM [Chen et al., 2010]. Ep-CAM is an oncogene that is upregulated in the presence of high EHMT2 expression. Another study suggests that the effects of EHMT2 on the development of breast cancer are via STAT3 activation and a subsequent reduction of microRNA-200c levels [Hua et al., 2014]. Finally, in AML, loss of EHMT2 expression significantly delays disease progression, presumably due to interaction of EHMT2, or lack thereof, with the transcription factor HOXA9 [Lehnertz et al., 2014].

In the present study, we have shown that EHMT2 is overexpressed in cervical cancer cells compared to normal cervical epithelial cells, suggesting that EHMT2 plays a similar role in cervical cancer as it does in a multitude of other cancers, namely that its presence promotes cell proliferation and the metastatic capacity of cervical cancer cells. We have then shown that if such overexpression is inhibited using interference RNA and knockdown of EHMT2, it reverses these tumorigenic effects while the apoptosis rate is increased, implying that EHMT2 inhibitors may offer an effective therapy for cervical cancer. Further, we have explained these effects by showing that inhibition of EHMT2 results in downregulated expression of tumorigenic proteins (Bcl-2, Mcl-1, and Survivin) involved in cell proliferation and viability and overexpression of the anti-malignant protein E-cadherin. Whether EHMT2 directly acts to induce expression of these tumorigenic proteins, such as by acting as a transcription factor or if the interaction is mediated by some downstream pathway, remains to be elucidated. Similarly, EHMT2, at least on some level, interacts with E-cadherin by directly preventing its transcription [Dong et al., 2012] or via a downstream pathway.

Recently, in vitro and in vivo studies by Chen et al. [2017] have shown that overexpression of EHMT2 in cervical cancer cell lines promoted cell proliferation and increased EHMT2-promoted migration and invasion in vitro. Therefore, combined with our findings, we propose that EHMT2 has a strong potential to serve as a therapeutic target, and the interference RNA shEHMT2 could be one such therapy. However, because the ability to direct interference RNA to specific tissues in the human body 
remains a pharmacologic challenge, other compounds that target EHMT2 could prove similarly effective.

Recent research has demonstrated that a novel peptide, A-366, which counteracts EHMT2 activity by competitive inhibition, can promote differentiation of leukemia cells in vitro and slow the growth of a leukemia xenograft in vivo [Pappano et al., 2015]. Another EHMT2 inhibitor, UNC0638, has been used to induce fetal hemoglobin expression as a potential therapy for sickle cell disease and beta thalassemia [Krivega et al., 2015]. While the efficacy of UNC0638 in cancer has not been evaluated, its mechanism of action suggests that it could be an effective therapy in EHMT2-implicated malignancies as well. BRD4770, a small molecule EHMT2 inhibitor, has been shown to have cytotoxic effects on pancreatic adenocarcinoma cells [Yuan et al., 2012, 2013]. Additionally, BIX01294, another EHMT2 inhibitor, has been shown to induce apoptosis in bladder cancer [Cho et al., 2011; Cui et al., 2015], neuroblastoma [Cui et al., 2015], oral squamous cell carcinoma [Ren et al., 2015], CML [Loh et al., 2014], and promyelocytic AML [Savickiene et al., 2014], 5 known EHMT2-mediated malignancies. Although UNC0321, an extremely potent EHMT2 inhibitor designed based on BIX-01294's structure, lacked sufficient cellular permeability, it led to the development of the more efficacious EHMT2 inhibitors UNC0646 and UNC0631 [Liu et al., 2011]. Finally, analogs of the com- pound sinefungin have been shown to be capable noncompetitive inhibitors of EHMT2 (as well as EHMT1) [Devkota et al., 2014]. Thus there are a handful of proteins that have the potential to serve as effective therapies for cervical cancer via EHMT2 inhibition.

In conclusion, our study confirmed the upregulation of EHMT2 in human cervical cancer cell lines. Also, our data suggest that EHMT2 plays a crucial role in cell proliferation and metastatic capacity in cervical cancer cells. We propose that EHMT2 could serve as a potential therapeutic target for cervical tumors.

\section{Acknowledgement}

The authors sincerely appreciate the infrastructure support of the Department of Central Laboratory of Shanghai Tenth People's Hospital.

\section{Statement of Ethics}

The authors have no ethical conflicts to disclose.

\section{Disclosure Statement}

The authors have no conflicts of interest to declare.

\section{References}

- Arbyn M, Castellsague X, de Sanjose S, Bruni L, Cho HS, Kelly JD, Hayami S, Toyokawa G, TakaSaraiya M, et al: Worldwide burden of cervical cancer in 2008. Ann Oncol 22:2675-2686 (2011).

Auclair G, Borgel J, Sanz LA, Vallet J, Guibert S, et al: EHMT2 directs DNA methylation for efficient gene silencing in mouse embryos. Genome Res 26:192-202 (2016).

-Candelaria M, de la Cruz-Hernandez E, TajaChayeb L, Perez-Cardenas E, Trejo-Becerril $C$, et al: DNA methylation-independent reversion of gemcitabine resistance by hydralazine in cervical cancer cells. PLoS One 7: e29181 (2012).

-Chen MW, Hua KT, Kao HJ, Chi CC, Wei LH, et al: H3K9 histone methyltransferase G9a promotes lung cancer invasion and metastasis by silencing the cell adhesion molecule EpCAM. Cancer Res 70:7830-7840 (2010).

-Chen RJ, Shun CT, Yen ML, Chou CH, Lin MC: Methyltransferase G9a promotes cervical cancer angiogenesis and decreases patient survival. Oncotarget 8:62081-62098 (2017). wa M, et al: Enhanced expression of EHMT2 is involved in the proliferation of cancer cells through negative regulation of SIAH1. Neoplasia 13:676-684 (2011).

Cui J, Sun W, Hao X, Wei M, Su X, et al: EHMT2 inhibitor BIX-01294 induces apoptosis through PMAIP1-USP9X-MCL1 axis in human bladder cancer cells. Cancer Cell Int 15: 4 (2015).

Devkota K, Lohse B, Liu Q, Wang MW, Staerk D, et al: Analogues of the natural product sinefungin as inhibitors of EHMT1 and EHMT2. ACS Med Chem Lett 5:293-297 (2014).

Ding J, Li T, Wang X, Zhao E, Choi JH, et al: The histone h3 methyltransferase G9a epigenetically activates the serine-glycine synthesis proliferation. Cell Metab 18:896-907 (2013).

Dong C, Wu Y, Yao J, Wang Y, Yu Y, et al: G9a interacts with snail and is critical for Snailmediated E-cadherin repression in human breast cancer. J Clin Invest 122:1469-1486 (2012). pathway to sustain cancer cell survival and
Heo K, Kim JS, Kim K, Kim H, Choi J, et al: Cellpenetrating $\mathrm{H} 4$ tail peptides potentiate p53mediated transactivation via inhibition of G9a and HDAC1. Oncogene 32:2510-2520 (2013).

Hua KT, Wang MY, Chen MW, Wei LH, Chen CK, et al: The H3K9 methyltransferase G9a is a marker of aggressive ovarian cancer that promotes peritoneal metastasis. Mol Cancer 13:189 (2014).

Ili CG, Brebi P, Lopez J, Garcia P, Leal P, et al: Genotyping of human papillomavirus in cervical intraepithelial neoplasia in a high-risk population. J Med Virol 83:833-837 (2011).

Kramer JM: Regulation of cell differentiation and function by the euchromatin histone methyltranserfases G9a and GLP. Biochem Cell Biol 94:26-32 (2016).

-Krivega I, Byrnes C, de Vasconcellos JF, Lee YT, Kaushal M, et al: Inhibition of G9a methyltransferase stimulates fetal hemoglobin production by facilitating LCR/gamma-globin looping. Blood 126:665-672 (2015). 
Lehnertz B, Pabst C, Su L, Miller M, Liu F, et al: The methyltransferase G9a regulates HOXA9-dependent transcription in AML. Genes Dev 28:317-327 (2014)

Liu F, Barsyte-Lovejoy D, Allali-Hassani A, He Y, Herold JM, et al: Optimization of cellular activity of G9a inhibitors 7-aminoalkoxy-quinazolines. J Med Chem 54:6139-6150 (2011).

Loh SW, Ng WL, Yeo KS, Lim YY, Ea CK: Inhibition of euchromatic histone methyltransferase 1 and 2 sensitizes chronic myeloid leukemia cells to interferon treatment. PLoS One 9:e103915 (2014).

Pappano WN, Guo J, He Y, Ferguson D, Jagadeeswaran S, et al: The histone methyltransferase inhibitor A-366 uncovers a role for G9a/GLP in the epigenetics of leukemia. PLoS One 10:e0131716 (2015).
Ren A, Qiu Y, Cui H, Fu G: Inhibition of H3K9 methyltransferase G9a induces autophagy and apoptosis in oral squamous cell carcinoma. Biochem Biophys Res Commun 459:1017 (2015).

Savickiene J, Treigyte G, Stirblyte I, Valiuliene G, Navakauskiene R: Euchromatic histone methyltransferase 2 inhibitor, BIX-01294, sensitizes human promyelocytic leukemia HL-60 and NB4 cells to growth inhibition and differentiation. Leuk Res 38:822-829 (2014).

-Shen XR, Feng R, Chai J, Cheng J, Wang DB: Modeling age-specific cancer incidences using logistic growth equations: implications for data collection. Asian Pac J Cancer Prev 15:9731-9737 (2014).

Wang SM, Qiao YL: Implementation of cervical cancer screening and prevention in China challenges and reality. Jpn J Clin Oncol 45: 7-11 (2015).
Wojtala M, Macierzynska-Piotrowska E, Rybaczek D, Pirola L, Balcerczyk A: Pharmacological and transcriptional inhibition of the G9a histone methyltransferase suppresses proliferation and modulates redox homeostasis in human microvascular endothelial cells. Pharmacol Res 128:252-263 (2018).

Yuan Y, Wang Q, Paulk J, Kubicek S, Kemp MM, et al: A small-molecule probe of the histone methyltransferase G9a induces cellular senescence in pancreatic adenocarcinoma. ACS Chem Biol 7:1152-1157 (2012)

Yuan Y, Tang AJ, Castoreno AB, Kuo SY, Wang Q, et al: Gossypol and an HMT G9a inhibitor act in synergy to induce cell death in pancreatic cancer cells. Cell Death Dis 4:e690 (2013).

Zhong X, Chen X, Guan X, Zhang H, Ma Y, et al: Overexpression of G9a and MCM7 in oesophageal squamous cell carcinoma is associated with poor prognosis. Histopathology 66:192-200 (2015) 